\title{
El rol del psicólogo en el síndrome de Tourette
}

\author{
Oriele Montezuma del Castillo
}

Lima - Perú

El artículo tiene el propósito de dar a conocer las diferentes áreas de actuación del psicólogo para el abordaje del Síndrome de Tourette. Se describe el síndrome en sus diferentes grados y su relación con otros desórdenes generalmente asociados. Se presentan algunos datos estadísticos de 21 personas con Sindrome de Tourette, los que son contrastados con otros estudios internacionales que consideraron estas variables. Se describen algunas características y consideraciones de importancia en el abordaje: a) individual de la persona con ST; b) grupal; c) de la familia; d) del entorno educativo y/o sociolaboral.

\section{Síndrome de Tourette / tics / rol del psicólogo / evaluación y terapia} cognitivo-conductual en el ST

\section{The roll of the psychologist in the Tourette syndrome}

The intention of this article is to present the different areas of psychologist performance for dealing with Tourette Syndrome. The syndrome is described in its varying degrees and its relationship with other generally associated disorders. Statistical data of 21 people with Tourette Syndrome, confronted with other international studies that considered these variables. Some characteristics and important considerations described in the treatment are: a) the individuality of the person with TS; b) groups; c)family; d) educative and/or socio labor environment. 
El desarrollo humano hace referencia a un maravilloso proceso de crecimiento que comienza desde que somos concebidos y finaliza con la muerte. Este proceso presenta una serie de características, a las que respondemos principalmente con admiración y luego con responsabilidad debido al reconocimiento de la influencia de nuestras acciones en la formación de seres humanos para que confíen en sí mismos y en su vida familiar y social, para que logren intervenir y responder funcionalmente en ese ambiente que ayudaron a crear, y se desempeñen de manera competente y disfruten plenamente de las aventuras que la vida les ofrece.

El síndrome de Tourette (ST), trastorno de la Tourette o de Gilles de la Tourette, es un desorden neurológico considerado crónico (tabla 1). Su principal característica es la ocurrencia de tics ${ }^{1}$ motores y vocales que pueden cambiar en localización y tipo, disminuir $\mathrm{y}$

1 Tic: "una vocalización o movimiento súbito, rápido, recurrente, no rítmico y estereotipado" (DSM-IV, 1995). "Sus componentes son: sintomas premonitorios (señales internas, sensaciones corporales localizadas 'tics sensitivos' y fenómenos evidentes (tics vocales o fónicos y motores), los que pueden ser simples o complejos. Conceptualmente, pueden ser concebidos como trozos de conductas preexistentes que pueden ser integradas como hábitos u otros actos voluntarios. $\mathrm{Al}$ igual que los hábitos, a menudo surgen de una sensibilidad aumentada y selectiva a señales del propio cuerpo o del exterior" (Leckman, J., 2002). aumentar en número, frecuencia, complejidad y severidad.

En su forma completa está acompañado de desórdenes que incluyen el trastorno por déficit de atención con hiperactividad (TDA/H), el trastorno obsesivo compulsivo (TOC) (figura 1) $\mathrm{y}$ una propensión a presentar problemas de conducta (PC), labilidad emocional, ansiedad, comportamiento oposicionista, depresión y, en ocasiones, problemas de aprendizaje.

Cuando el ST es incompleto o en un grado de severidad muy leve, generalmente no requiere de tratamiento o intervención psicológica alguna. Se estima que entre el $30 \%$ y $40 \%$ tienen síntomas que desaparecen en la última etapa de la adolescencia. Sin embargo, cuando se presenta en su forma completa $\mathrm{y} / \mathrm{o}$ el grado de severidad es mayor a leve, sus manifestaciones influyen de manera directa o indirecta en el desarrollo del niño, tiñendo $y$ moldeando su interacción con el medio (King, 1999). En estos casos, un programa interdisciplinario y el esfuerzo coordinado de profesionales: médico, maestros, psicólogo, terapeutas del lenguaje y aprendizaje, entre otros, de la familia y de la propia persona con ST, según las necesidades, son cruciales para el futuro de ésta.

El trabajo en equipo presenta una serie de retos y desafíos, pero se puede afirmar que en nuestro país, para el abordaje de las personas que presentan el ST, es una realidad, que se traduce 


\section{Tabla No 1}

\section{Trastorno de la Tourette}

A. En algún momento, a lo largo de la enfermedad, ha habido tics motores múltiples y uno o más tics vocales, aunque no necesariamente al mismo tiempo.

B. Los tics aparecen varias veces al día (habitualmente en oleadas) casi todos los días o intermitentemente por más de un año y durante este tiempo nunca hay un período libre de tics superior a los tres meses consecutivos.

C. El trastorno provoca un notable malestar o deterioro significativo social y laboral o de otras áreas importantes de la actividad del individuo.

D. El inicio es anterior a los 18 años.

E. La alteración no se debe a los efectos fisiológicos directos de un fármaco (por ejemplo estimulantes) o de una enfermedad médica (por ejemplo Huntington o encefalitis posvírica).

\section{Figura $\mathbf{N}^{\circ} 1$ \\ Espectro clínico del Síndrome de Tourette*}

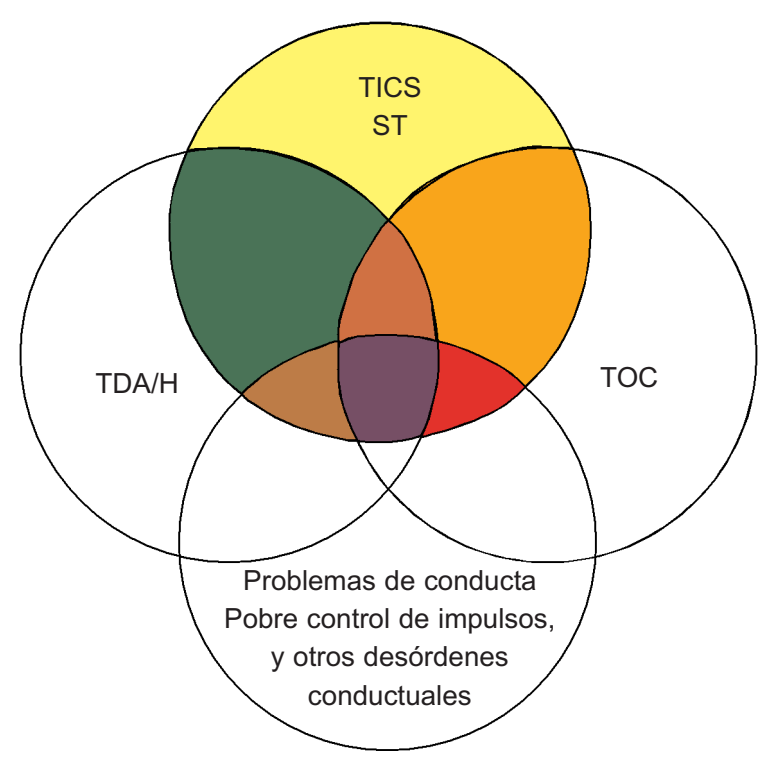

* Filomeno, 1994; Leckman, 2002, modificado Montezuma, O. (ST = Síndrome de Tourette, TDA/H = trastorno por déficit de atención con hiperactividad, TOC = trastorno obsesivo compulsivo). 
en la participación abierta de los profesionales, familiares y amigos que desde 1992 conforman los diferentes grupos de la Asociación Síndrome de Tourette del Perú (ASTP). A ello se agrega la disposición de numerosos maestros que se han interesado en el síndrome y que lo han asumido como una suerte de reto en sus centros educativos, regulares y especializados abriendo sus puertas y brindando sus recursos para su abordaje integral. La participación del psicólogo en los equipos así formados, puede reconocerse de suma importancia, con frecuencia es gestor, promotor $y$ soporte de éste.

La labor del psicólogo tiene diferentes áreas, etapas y formas de acción. Participa activamente en la prevención, evaluación, diagnóstico, tratamiento e integración de la persona con ST, así como puede y debe aportar datos de investigación; acciones que trataremos de revisar, no sin antes señalar que requiere de una preparación especial, profunda y comprometida así como una discusión periódica con la persona que padece el ST y su familia sobre la aceptación y pertinencia de las diferentes maneras utilizadas para abordar los problemas, ya que cada persona con ST es diferente, única, con una variedad de factores singulares que determinan su comportamiento individual y el aumento y disminución de sus síntomas.

La investigación acerca del ST es extensa y los datos que se han publicado señalan una serie de patrones que es importante conocer antes de la intervención psicológica pertinente. En este trabajo se presentan algunas estadísticas de 21 casos de personas con ST (tabla 2), de las 86 que han realizado algún contacto con la ASTP, las que serán contrastadas con otros estudios internacionales que consideraron las variables analizadas. Es importante señalar que el grupo trabajado presenta características particulares que debemos tomar en cuenta a la luz de los resultados, principalmente porque los síntomas conductuales sufren cambios importantes a lo largo del desarrollo: son niños entre los 4 y 15 años (tabla 3 ) que llegaron al consultorio con un ST de moderado a muy severo $(80,95 \%)$ y asociados a otros trastornos como el TDA/H $(76,19 \%$ de los casos) y/o al TOC $(61,90 \%)$ (tablas 4 y 5 ).

Tabla $\mathbf{N}^{\circ} 2$

\begin{tabular}{lcr}
\multicolumn{3}{c}{$\begin{array}{c}\text { Frecuencia (\%) según el sexo de } 21 \\
\text { personas con ST }\end{array}$} \\
\hline Sexo & Hombres & Mujeres \\
\hline f. & 16 & 5 \\
$\%$ & 76,19 & 23,81 \\
\hline
\end{tabular}

\section{Tabla $N^{\circ} 3$}

Distribución por edades de 21 personas con ST

\begin{tabular}{lcccc}
\hline Edad & 4 a 5 & 5 a 7 & 8 a 11 & 12 a 15 \\
\hline f. & 1 & 4 & 10 & 6 \\
$\%$ & 4,76 & 19,05 & 47,62 & 28,57 \\
\hline
\end{tabular}


Tabla No 4

Frecuencia (\%) del grado de severidad de 21 personas con ST

\begin{tabular}{ccccccc}
\hline & Muy leve & Leve & Moderado & Fuerte & Severo & Muy severo \\
\hline$f$ & 2 & 2 & 7 & 4 & 5 & 1 \\
$\%$ & 9,52 & 9,52 & 33,33 & 19,05 & 23,8 & 4,76
\end{tabular}

Tabla $\mathrm{N}^{\circ} 5$

Frecuencias (\%) de los problemas asociados en 21 personas con ST

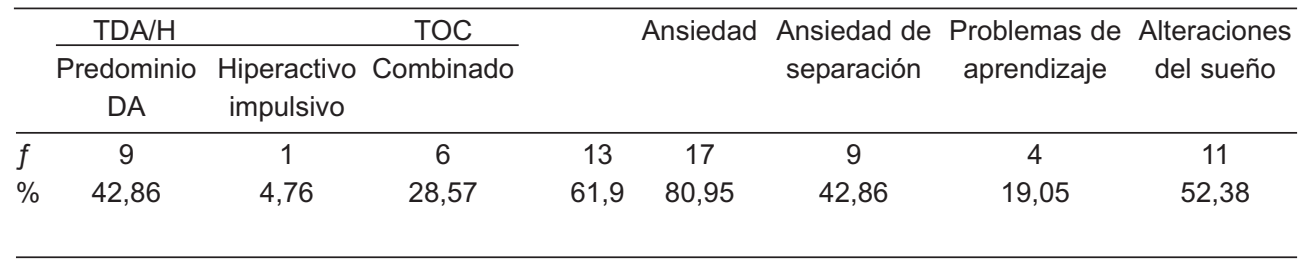

\section{DATOS DE IMPORTANCIA}

\section{Frecuencia del STy diferencias de género}

Según los estudios se estima que la frecuencia del ST es de 1 entre 100 en niños y de 1 entre 600 en niñas, (una relación de 6 niños por 1 niña) si se consideran las formas incompletas y en sus formas completas 1 de cada 2.500 personas (Tourette Association, NY). En la tabla 6, que considera las 86 personas que se contactaron con la ASTP, la relación en frecuencia es de 5 (hombres) a 1 (mujeres) según el género.
Tabla $\mathrm{N}^{\circ} 6$

Frecuencia (\%) de personas con ST, según sexo, que contactaron con la ASTP*

\begin{tabular}{lccc}
\hline & Hombres & Mujeres & Total \\
\hline$f$ & 72 & 14 & 86 \\
$\%$ & 83,72 & 16,28 & 100 \\
\hline Relación & 5 hombres por 1 mujer & \\
\hline
\end{tabular}

*Asociación Síndrome de Tourette del Perú

Edad de aparición de la sintomatología para el diagnóstico del ST

La aparición de la sintomatología que permite diagnosticar a un niño con el ST, se presenta aproximadamente a los 6 años y medio de edad. Como se 
puede observar en la tabla 7 , la edad media oscila entre los 5,8 y los 7 años. En nuestro grupo las cifras son similares, en el $57,14 \%$ de los sujetos la edad de aparición se encuentra entre los $5 \mathrm{y}$ los 7 años (figura 2).

Tabla No 7

Edad media de inicio de los tics según diversos autores (entre paréntesis, número de casos).

\begin{tabular}{ll}
\hline Investigadores edad de inicio & \\
\hline Brunn (n: 350) & 5,8 \\
Erenberg y Rothner (n: 200) & 6,3 \\
Freeman et al. (n: 3,500) & 6,4 \\
Shapiro et al. (n: 661) & 6,7 \\
Coming y Coming (n: 250) & 6,9 \\
Goldenberg et al. (n: 112) & 6,9 \\
Nee et al. (n: 50) & 7,0 \\
Lees et al (n: 53) & 7,0 \\
\hline
\end{tabular}

Fuente: Fernández, 2002.

Figura $\mathrm{N}^{\circ} 2$

Histograma de edad de aparición de tics en 21 casos con ST

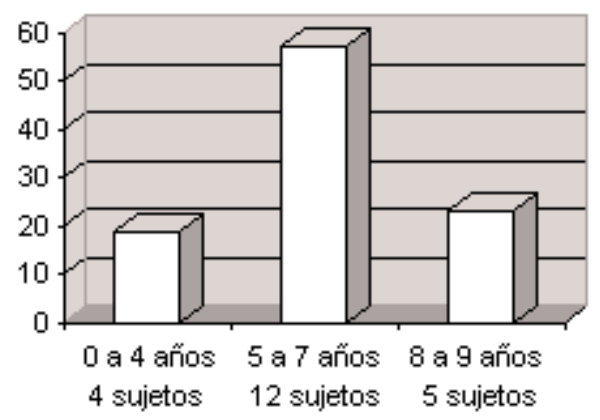

\section{Antecedentes familiares identificados}

Actualmente es claro que el ST es un desorden genético, que la vulnerabilidad (el niño recibe la base genética o constitucional para desarrollar el desorden) al ST es transmitida de generación en generación, y que hay diferentes formas en que se puede expresar esa vulnerabilidad, las que incluyen el ST completo, tics múltiples crónicos y últimamente el reconocido TOC (Dowling, 1995). Los estudios de familias señalan que el $44 \%$ de pacientes con tics presentan una historia familiar positiva de tics, y el $30 \%$ de signos positivos de TOC. En el grupo analizado son, efectivamente, estos antecedentes los que se detectaron en algún miembro de la familia. En la tabla 8 se observa que en el $66,67 \%$ se encontró la presencia de tics y en el $61,9 \%$ el TOC.

Tabla $N^{\circ} 8$

\begin{tabular}{ccc}
$\begin{array}{c}\text { Frecuencias (\%) de los principales } \\
\text { antecedentes familiares de } \\
\text { 21 personas con ST }\end{array}$ \\
\hline Tics & TOC & Otros \\
\hline 14 & 13 & 7 \\
66,67 & 61,09 & 33,33 \\
\hline
\end{tabular}

\section{Diagnósticos antes del ST}

Se ha reportado que antes del diagnóstico del ST, los niños generalmente recibieron otros diagnósticos, el más común de los cuales es el TDA/H, problemas de conducta o TOC (Comings, 1990). En la tabla 9 se observa que el 
$19,05 \%$ de niños con los que se trabajó habían recibido el diagnóstico de $\mathrm{TDA} / \mathrm{H}$, el $14,29 \%$ el de problemas de conducta (PC), el 38,10\% como TDA/H y problemas de conducta, el 9,52\% ningún diagnóstico, el 9,52\% $\mathrm{TDA} / \mathrm{H}, \mathrm{PC}$ y otros trastornos asociados, y el 4,76\% TDA y otros.

Tabla $\mathrm{N}^{\circ} \mathrm{9}$

Frecuencias (\%) de los diagnósticos recibidos por 21 personas antes del de ST

\begin{tabular}{lcc}
\hline & $f$ & $\%$ \\
\hline TDA/H & 4 & 19,05 \\
Problema de & & \\
conducta (PC) & 3 & 14,29 \\
Otros & 1 & 4,76 \\
Ninguno & 2 & 9,52 \\
TDA/H y PC & 8 & 38,10 \\
TDA-PC y otros & 2 & 9,52 \\
TDA y otros & 1 & 4,76 \\
\hline
\end{tabular}

Asimismo, se encuentra que el diagnóstico más común emitido antes del de Tourette fue el de TDA/H, acompa- ñado de problemas de conducta (correlación positiva en la tabla 10) pero no si éstos se presentan aislados (correlación negativa, tablas 11 y 12).

Como se anotó anteriormente, el ST se encuentra asociado a otros trastornos, también conocidos como trastornos comórbidos. En la tabla 13 se presentan las frecuencias en términos de porcentajes de los principales estudios realizados en relación con los hallazgos en el grupo trabajado (FernándezÁlvarez, 2002; 1992). En nuestro grupo, el $76,19 \%$ presentó alguno de los tipos de TDA/H, de éstos el $42,86 \%$ con predominio del déficit de atención, el $4,76 \%$ con predominio hiperactivoimpulsivo y el $29,57 \%$ de tipo combinado. El 61,9\% presentó el TOC asociado, el 19,05\% presentó problemas de aprendizaje, el 80,95\% ansiedad, el $42,86 \%$ ansiedad de separación y el $52,38 \%$ alteraciones del sueño. Las diferencias en relación con los hallaz-

Tabla $N^{\circ} 10$

Relación entre el diagnóstico inicial de TDA/H y PC con el diagnóstico de ST

Correlación

\begin{tabular}{|c|c|c|c|c|}
\hline & & & $\begin{array}{l}\text { Síndrome de } \\
\text { Tourette }\end{array}$ & $\begin{array}{l}\text { Diagnóstico } \\
\text { TDA/H y PC } \\
\text { antes de ST }\end{array}$ \\
\hline \multirow[t]{3}{*}{ Spearman's } & Síndrome de & Correlación & 1.000 & $.469^{*}$ \\
\hline & Tourette & Sig. (2 colas) & . & .032 \\
\hline & & $\mathrm{N}$ & 21 & 21 \\
\hline
\end{tabular}

\footnotetext{
* La correlación es significativa en el nivel .05.
} 
Tabla No 11

Relación entre el diagnóstico inicial de TDA/H con el diagnóstico de ST

Correlación

\begin{tabular}{ccccc}
\hline & & Síndrome de \\
Tourette & & $\begin{array}{c}\text { Diagnóstico } \\
\text { TDA/H y PC } \\
\text { antes de ST }\end{array}$ \\
\hline Spearman's & Diagnóstico & Correlación & 1.000 & $-.538^{*}$ \\
& TDA/h antes de & Sig. (2-tailed) &. & .012 \\
ST & $\mathrm{N}$ & 21 & 21 \\
\hline
\end{tabular}

* La correlación es significativa en el nivel .05.

Tabla No 12

Relación entre el diagnóstico inicial de problemas de conducta con el diagnóstico de ST trastornos conductuales asociados al ST

Correlación

\begin{tabular}{|c|c|c|c|c|}
\hline & & & $\begin{array}{l}\text { Síndrome de } \\
\text { Tourette }\end{array}$ & $\begin{array}{l}\text { Diagnóstico } \\
\text { TDA/H y PC } \\
\text { antes de ST }\end{array}$ \\
\hline Spearman's & $\begin{array}{l}\text { Síndrome de } \\
\text { Tourette }\end{array}$ & $\begin{array}{c}\text { Correlación } \\
\text { Sig. (2 colas) } \\
\mathrm{N}\end{array}$ & $\begin{array}{c}1.000 \\
\cdot \\
21\end{array}$ & $\begin{array}{c}-.209 \\
.363 \\
21\end{array}$ \\
\hline
\end{tabular}

gos en otros reportes pueden ser explicadas por las características de los sujetos analizados, ya que éstos asisten a consulta debido a la presencia interferente de los trastornos asociados y por el número de sujetos trabajados.

\section{Hipersensibilidad}

Las personas con ST presentan no sólo tics motores y vocales sino también lo que se ha venido a llamar tics sensitivos, muy difíciles de reconocer y que son descritos por ellos mismos como una sensación incómoda e imprecisa de presión, irritación, calor, frío, etc., que las lleva a realizar movimientos o sonidos (Kurlan, 1989). Algunas personas con ST presentan una hipersensibilidad no sólo táctil (no soportan la presión sobre la piel, como ropa ajustada) sino olfativa (son capaces, en algunos casos, de identificar muy finamente los olores), y esta agudeza los perturba. También refieren ocasionalmente hipersensibilidad a los sonidos. En la tabla 14 se presenta la frecuencia en que ésta aparece en las 21 personas analizadas (el 71\% manifestó una hipersensibilidad táctil y el $42 \%$ olfativa). 
Tabla $\mathbf{N}^{\circ} 13$

Frecuencia (\%) de algunos trastornos asociados en sujetos con ST

\begin{tabular}{|c|c|c|c|c|c|c|c|}
\hline & $\begin{array}{c}\text { Commins } \\
1995 \\
(n: 353)\end{array}$ & $\begin{array}{l}\text { Freeman } \\
\text { et al } \\
(n: 3,500)\end{array}$ & $\begin{array}{c}\text { Fdes-Alvarez } \\
2002 \\
\text { (n: 219) }\end{array}$ & $\begin{array}{c}\text { Chee,Ky } \\
\text { et al } \\
1992\end{array}$ & $\begin{array}{c}\text { Robertson, } \\
\text { et al } \\
1988\end{array}$ & $\begin{array}{l}\text { Coffey } \\
\text { et al } \\
1992 \\
(n: 84)\end{array}$ & $\begin{array}{c}\text { Montezuma* } \\
2003 \\
(\mathrm{n}: 21)\end{array}$ \\
\hline $\mathrm{TDA} / \mathrm{H}$ & 60.9 & 60 & 42 & 32 & & & 76.19 \\
\hline TOC & 57.5 & 59 & 45 & & 40 & 30 & 61.9 \\
\hline $\begin{array}{l}\text { Problemas de } \\
\text { aprendizaje }\end{array}$ & 37.4 & 23 & & & & & 19.05 \\
\hline $\begin{array}{l}\text { Ansiedad } \\
\text { Ansiedad de }\end{array}$ & & & & 30 & & & 80.95 \\
\hline $\begin{array}{l}\text { separación } \\
\text { Alteraciones } \\
\text { del sueño }\end{array}$ & 56.1 & 25 & & & & 36 & 42.86 \\
\hline
\end{tabular}

* Datos encontrados en 21 sujetos con ST estudiados en Lima.

Tabla No 14

Frecuencias (\%) de personas que presentan hipersensibilidad táctil y/u olfativa en 21 personas con ST

\begin{tabular}{lcc}
\hline & Táctil & Olfativa \\
\hline$f$ & 15 & 9 \\
$\%$ & 71,43 & 42,86 \\
\hline
\end{tabular}

\section{Requerimientos de educación especializada}

Se afirma que sólo el $30 \%$ de los niños con ST tienen serios problemas escolares y requieren de educación especializada. (Asociación Americana del Síndrome de Tourette) aunque en el estudio de Abwender et al., 1996, (citado en Leckman, 1999), el 46\% de las personas con ST sufrían dificultades relacionadas con la escolaridad. En la tabla 15 observamos que en los sujetos analizados el 19,04\% requirieron edu- cación especializada, de los cuales el $9,52 \%$ se incorporaron a esta modalidad de educación desde el tercer grado, el 9,52\% desde secundaria, mientras que el $80,95 \%$ permanecieron recibiendo educación regular.

Los sujetos de nuestro grupo recibieron terapia desde la aparición de la primera sintomatología, lo que puede haber tenido un efecto beneficioso sobre el rendimiento escolar. De los ocho sujetos que se presentaron a la educación superior el $75 \%$ ingresaron; se mantienen estudiando el 50\% de ellos. El bajo rendimiento escolar o la necesidad de educación especializada se ha vinculado a las manifestaciones del TDA/H; del TOC u otras dificultades neuropsicológicas a las que los niños con ST presentan mayores riesgos (Schultz et al., 1998). 
Tabla $\mathbf{N}^{\circ} 15$

Frecuencias (\%) de personas con ST

en relación con el tipo de

educación recibida

\begin{tabular}{lccccc}
\hline Etapa & \multicolumn{3}{c}{ Escolar } & \multicolumn{2}{c}{ Superior } \\
\hline Educación & Regular & $\begin{array}{c}\text { Especializada } \\
\text { desde } 3^{\circ}\end{array}$ & $\begin{array}{c}\text { Especializada } \\
\text { desde }\end{array}$ & Ingresó de 8 & $\begin{array}{c}\text { Se } \\
\text { mantiene }\end{array}$ \\
\hline$f$ & 17 & 2 & 2 & 6 & 4 \\
$\%$ & 80,95 & 9,52 & 9,52 & 75 & 50 \\
\hline
\end{tabular}

\section{Cocientes de inteligencia}

Se afirma que el cociente de inteligencia de los niños con ST no es diferente al resto de la población, y esto se comprueba en los sujetos estudiados. Como se aprecia en la tabla 16, todas las puntuaciones -obtenidas de la evaluación con las diferentes escales del Wechslerse ajustan a una distribución normal, tanto en los subtests como los puntajes verbal (PTV), ejecutivo (PTE) y total (PTG). En este caso se trabajaron sólo con 18 de los sujetos debido a que no se contaban con todos los datos requeridos para los tres sujetos restantes.

Tabla No 16

Prueba de Bondad de Ajuste de Kolmogorov-Smirnov para la distribución de subtests y puntajes del Wechsler en 21 personas con ST

\begin{tabular}{lccccccccccccc}
\hline & $\mathrm{I}$ & $\mathrm{S}$ & $\mathrm{A}$ & $\mathrm{V}$ & $\mathrm{C}$ & $\mathrm{RD}$ & $\mathrm{CF}$ & $\mathrm{AF}$ & $\mathrm{DB}$ & $\mathrm{EO}$ & $\mathrm{PTV}$ & $\mathrm{PTE}$ & $\mathrm{PTG}$ \\
\hline $\mathrm{N}$ & 18 & 18 & 18 & 18 & 18 & 18 & 18 & 18 & 18 & 18 & 18 & 18 & 18 \\
\hline Media & 16.0 & 16.11 & 14.72 & 29.89 & 18.06 & 11.61 & 17.94 & 23.00 & 31.72 & 25.56 & 106.39 & 98.22 & 204.61 \\
D.E. & 5.98 & 5.82 & 4.25 & 11.58 & 5.79 & 3.31 & 4.73 & 14.02 & 13.35 & 6.97 & 32.73 & 33.75 & 63.41 \\
$\mathrm{~K}-\mathrm{S}$ & .517 & .632 & .582 & .691 & .625 & .905 & .491 & .595 & .456 & .610 & .359 & .608 & .405 \\
Z & & & & & & & & & & & & & \\
Sig. & .952 & .820 & .887 & .726 & .830 & .385 & .969 & .871 & .985 & .850 & 1.000 & .854 & .997 \\
\hline \multicolumn{1}{l}{ p.<.05 } & & & & & & & & & & & & &
\end{tabular}

a: La distribución del test es normal

b: Cálculo de los datos

$\mathrm{I}=$ Información, $\mathrm{S}=$ Semejanzas, $\mathrm{A}=$ aritmética, $\mathrm{V}=$ vocabulario, $\mathrm{C}=$ comprensión, $\mathrm{RT}$ = retención de dígitos, $\mathrm{CF}$

= completamiento de figuras, $\mathrm{AF}=$ arreglo de figuras, $\mathrm{DB}=$ diseño con bloques, $\mathrm{EO}=$ ensamblaje de objetos, $\mathrm{PTV}$

= puntaje verbal, PTE = puntaje ejecutivo, PTG = puntaje global. 
Mejor rendimiento en el área verbal que en la ejecutiva y su relación con los trastornos asociados

Se ha encontrado que los sujetos con ST rinden mejor en el área verbal (PTV) que en la ejecutiva (PTE). También se afirma que el rendimiento alterado en el puntaje ejecutivo se correlaciona con la presencia de síntomas del TOC más el TDA/H y el TOC solo, pero no cuando se presenta el TDA/H solo. En los sujetos trabajados comprobamos que hay diferencia en el rendimiento entre el PTV y el PTE, la que es una diferencia significativa (tabla 17), y que en los sujetos que presentan TOC esta diferencia también es significativa (tabla 18).

\section{Relaciones entre otros trastornos asociados}

Otros hallazgos en los 21 sujetos trabajados son: existe una relación significativa entre la presencia de episodios de ansiedad de separación en la historia de los sujetos con ST, con la presentación de alteraciones del sueño (tabla 19). Igualmente, una relación significativa entre estos episodios de ansiedad de separación con el TOC mas no con el TDA/H; en este último caso la correlación es negativa, esto es, las personas con ST que han presentado ansiedad de separación no son precisamente las que tienen el TDA/H (tablas 20 y 21 ) y que la presencia de trastornos de ansiedad se asocia al grado de severidad del ST (figura 3).

Tabla $N^{\circ} 17$

Diferencia de medias entre el puntaje verbal y el ejecutivo en los sujetos con ST

\begin{tabular}{lcccc}
\hline & Media & D.E & $t$ & Sig \\
\hline PTV & 106.39 & 32.73 & 13.790 & $.000^{\star *}$ \\
PTE & 98.22 & 33.75 & 12.348 & $.000^{\star *}$ \\
\hline
\end{tabular}

${ }^{* *}$ p. $<.01$

Tabla $\mathbf{N}^{\circ} 18$

Diferencia de medias entre el puntaje verbal y el ejecutivo en los sujetos con ST y TOC

\begin{tabular}{lcccc}
\hline & Media & D.E & $\mathrm{t}$ & Sig \\
\hline PTV & 107.00 & 36.49 & 10.571 & $.000^{* *}$ \\
PTE & 100.38 & 37.38 & 9.684 & $.000^{* *}$ \\
\hline
\end{tabular}

${ }^{* *} \mathrm{p} .<.01$ 
Tabla No 19

Relación entre alteraciones del sueño y ansiedad de separación en 21 personas con ST

\begin{tabular}{lllcc}
\hline & & Alteraciones & & $\begin{array}{c}\text { Presentó } \\
\text { del sueño } \\
\text { ansiedad de } \\
\text { separación }\end{array}$ \\
\hline Spearman's & Alteraciones & Correlación & 1.000 & $.440^{*}$ \\
& del sueño & Sig. (2 colas) &. & .046 \\
& $\mathrm{n}$ & 21 & 21 \\
\hline
\end{tabular}

* La correlación es significativa en el nivel .05.

Tabla $\mathbf{N}^{\circ} 20$

Relación entre ansiedad de separación y ST con TOC en 21 personas con ST

\begin{tabular}{lllcc}
\hline & & $\begin{array}{c}\text { Presentó } \\
\text { ansiedad de } \\
\text { separación }\end{array}$ & $\begin{array}{c}\text { ST } \\
\text { asociado a } \\
\text { TOC }\end{array}$ \\
\hline Spearman's & Presentó & Correlación & 1.000 & $.548^{*}$ \\
& ansiedad de & Sig. (2 colas) &. & .010 \\
& separación & $\mathrm{n}$ & 21 & 21 \\
\hline
\end{tabular}

* La correlación es significativa en el nivel .05.

Tabla No 21

Relación entre ansiedad de separación y ST con TDA/H

\begin{tabular}{lllcc}
\hline & & $\begin{array}{c}\text { Presentó } \\
\text { ansiedad de } \\
\text { separación }\end{array}$ & $\begin{array}{c}\text { ST } \\
\text { asociado a } \\
\text { TDA/H }\end{array}$ \\
\hline Spearman's & Presentó & Correlación & 1.000 & -.084 \\
& ansiedad de & Sig. (2 colas) &. & .718 \\
& separación & $\mathrm{n}$ & 21 & 21 \\
\hline
\end{tabular}


Figura $N^{\circ} 3$

Grados de severidad del síndrome con relación a los trastornos de ansiedad en 21 personas con ST

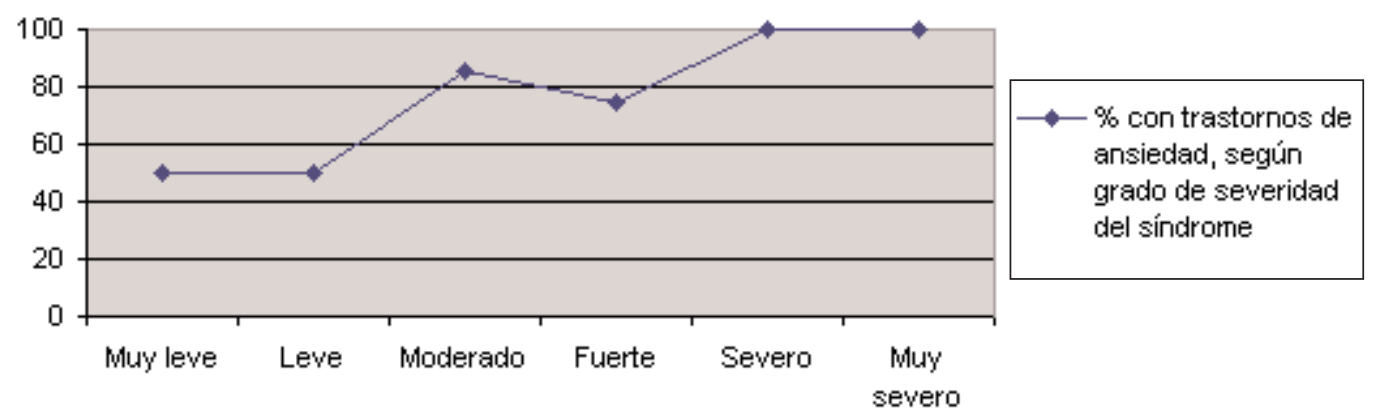

EVALUACIÓN Y TERAPIA PSICOLÓGICA DEL PACIENTE CON ST

A pesar de que el ST tiene una etiología primaria neurobiológica, los factores psicosociales pueden influenciar, de manera decisiva, en el curso del desarrollo, la severidad y la manifestación del trastorno. Hay abundante evidencia de que estresores no específicos, así como factores psicológicos familiares e individuales, pueden aminorar o exacerbar los síntomas y producir cambios mensurables (King et al., 1999). Hay factores individuales, familiares y culturales que moldean la manera en que las personas con ST, su familia, los padres y profesores perciben, dan significado y responden a los síntomas. Factores psicosociales que influyen en la manera como la persona con ST y su familia se aproxima a los diferentes tipos de intervención, incluyendo la medicación. Es aquí, en los niveles individual y relacional, donde el psicólogo puede influir de manera determinante.

La acción del psicólogo, así como de los otros profesionales, se ve influenciada por el momento de evolución en la que se encuentra la presentación del síndrome. Podemos diferenciar tres etapas: a) el inicio o momento de emergencia, con síntomas que pueden corresponder o no al síndrome; b) el intermedio, en el que aparece más claramente la sintomatología, con sus movimientos, cambios, inestabilidades y las diferentes trayectorias que éstas siguen, y c) el final, o el establecimiento del grado de severidad real del síndrome (Bliss, 1980).

Reconocer el inicio del trastorno, analizarlo y estudiarlo, compartir los cuestionamientos con el equipo, mantenerse en espera y, si es posible, desterrarlo, son momentos terriblemente difíciles para el psicólogo; y, lo más importante, los esfuerzos son sólo exito- 
sos intermitentemente, por lo que tenemos que elegir entre dejar todo "colgado", evadir el tema o seguir investigando de manera compartida para llegar a una conclusión.

Es importante resaltar que la descripción de los primeros síntomas es realizada por los padres y que con cierta frecuencia, cuando se cuenta con una información masiva, como la que trata de realizar en nuestro país la ASTP, es la propia familia o sus allegados los que diagnostican el síndrome. En la tabla 22 se aprecia que en nuestro medio el $19,05 \%$ de los casos fueron identificados por algún miembro de la familia, el 9,52\% por sus maestros, y el $71,43 \%$ por médicos especializados (neurólogos o psiquiatras) o psicólogos. Esto podría explicarse porque la ASTP tiene sólo cinco años de constituida, a diferencia de la Asociación Americana, que contribuye con fondos para investigaciones importantes en diversas áreas relevantes desde 1984, y que ha difundido las características del síndrome de manera masiva hace más de tres décadas.

Tabla $\mathbf{N}^{\circ} 22$

Frecuencia (\%) de los que identificaron el trastorno, en 21 personas con ST

\begin{tabular}{|c|c|c|c|}
\hline & Familia & Maestros & $\begin{array}{c}\text { Médico } \\
\text { Neurólogo } \\
\text { Psicólogo } \\
15\end{array}$ \\
\hline$\%$ & 19,05 & 9,52 & 71,43 \\
\hline
\end{tabular}

En cuanto a la evaluación y tratamiento de la persona con ST y su familia el abordaje puede ser individual y grupal, como se puede apreciar en la figura 4.

\subsection{Abordaje individual}

\subsubsection{Evaluación psicológica general}

Consiste en realizar un análisis descriptivo (o topográfico) y un análisis funcional (proceso continuo y constante de investigación, con su debida formulación y contrastación de hipótesis) donde se debe poner atención al medio externo y al mundo interno que circundan la conducta, en todos sus aspectos: físicos, fisiológicos, cognitivos, sociales y emocionales, entre otros, que permita el posterior diseño de intervención y el seguimiento pertinente.

En esta evaluación podemos distinguir los siguientes objetivos:

- Análisis funcional de conductas.Discriminar, analizar y describir operativamente conductas que pueden ser categorizadas como excesos o déficits, y si es preciso evaluar el peso, y establecer la línea base de cada componente del síndrome, así como los efectos colaterales que los fármacos pudieran estar produciendo en los diferentes momentos del proceso, en cada caso.

- Monitorear las fluctuaciones de los sintomas en los contextos especificos de su emergencia, en situacio- 


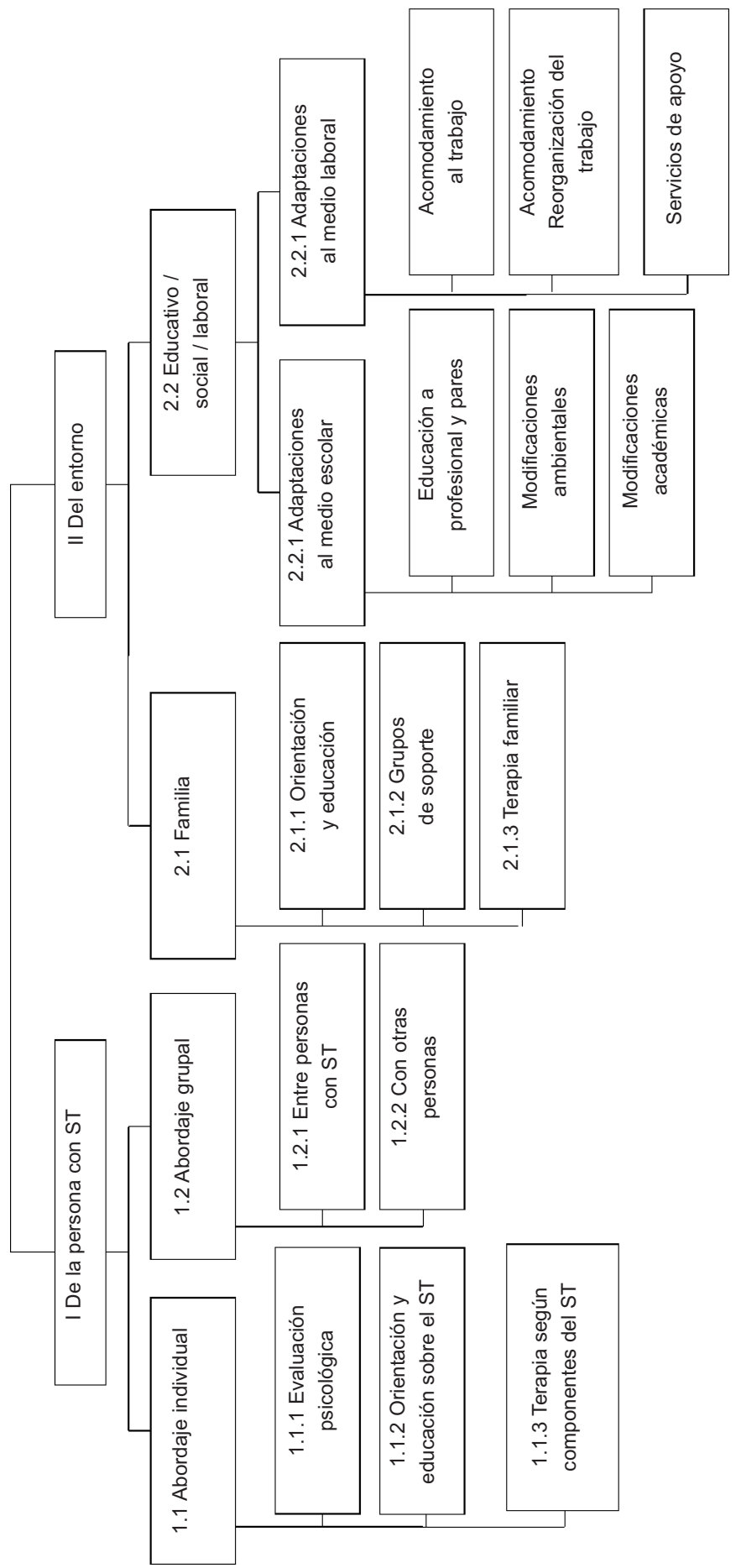


nes estresantes o en la vida diaria.Los síntomas del ST pueden ser catalogados de muy leves, leves, moderados, fuertes, severos y muy severos, por su frecuencia, su complejidad y el grado en que ellos causan interferencia en las actividades de la vida diaria y las actividades básicas cotidianas de la persona con ST. Lo que puede ser medido por varias escalas, entre las cuales de encuentra la escala de severidad del ST de Shapiro y sus colaboradores, 1989 (Bados, 1999). Es conocido que los pacientes pueden inhibir los síntomas, o como ellos mismos mencionan, no sentir la urgencia de emitirlos mientras están en el consultorio médico, el colegio o el trabajo. Sin embargo, en otros ambientes, como el hogar o el consultorio psicológico, emergen, se disparan con violencia y cambian en intensidad y ubicación, presentando oscilaciones, es por eso necesario el registro de estas conductas a través de métodos de evaluación conductual en ambientes naturales.

- La descripción y evaluación de las fortalezas y debilidades de las personas que presentan el ST.- En los aspectos cognitivos y socioemocionales, la evaluación debe incluir:

- El cociente de inteligencia, que, como hemos visto, no es diferente al del resto de la población; sin embargo, generalmente presentan mejor rendimiento en el área verbal que en el área ejecutiva.

- Las características de la atención, tanto en la sostenida como en la dividida. Se pueden apreciar dificultades y una gran influencia en las otras áreas de desarrollo, como la interferencia en el proceso enseñanza-aprendizaje.

- El rendimiento en el aspecto psicomotor, en algunos casos se detectan dificultades en la coordinación motora fina, organización espacial, disociación del movimiento, disturbios en el esquema e integración del control motor. En la tabla 23 se encuentra que el $71,43 \%$ de los sujetos con ST presentaron bajo rendimiento en coordinación motora fina y el $38 \%$ en coordinación motora gruesa.

Tabla $\mathbf{N}^{\circ} 23$

Frecuencia (\%) con bajo rendimiento en coordinación motora fina y gruesa en 21 personas con ST

\begin{tabular}{lcc}
\hline & CMF* $^{*}$ & CMG $^{* *}$ \\
\hline$f$ & 15 & 8 \\
$\%$ & 71,43 & 38,1 \\
\hline \multirow{2}{*}{ " CMF = coordinación motora fina, ${ }^{* *} \mathrm{CMG}=$ coordi- } \\
nación motora gruesa
\end{tabular}

- El estado de alerta. Se encuentra con frecuencia un estado que puede ser excesivo y que se manifiesta con irritabilidad, agresividad, baja tolerancia a la frustración, ansiedad, exabruptos, 
hipersensibilidad, dificultad para planear, incapacidad para terminar el trabajo, bajo autocontrol, invasión de tiempo y espacio o bajo, que se manifiesta con una disminución de la motivación; un locus de control generalmente externo, dificultades para evaluar y solucionar problemas, bajo procesamiento de la información, estrategias de aprendizaje globales, holísticas, dependencia de campo. Este estado de alerta en las personas con ST varía de un extremo al otro de manera muy inestable.

Estos hallazgos son datos de línea base que pueden servir para evaluar el efecto de algunos fármacos y aclarar la presencia de otros problemas asociados, para la programación de una terapia correctiva y/o prever los ajustes ambientales escolares y/o laborales necesarios.

- Identificar las características funcionales y dinámica familiar (modelos, detección de líderes, niveles de aceptación entre los miembros, incluyendo las culpas por el origen genético del síndrome, las respuestas que los padres dan a "sus rarezas"...). La secuela emocional de la acción de la familia puede afectar al paciente más allá de su niñez.

- Analizar la ubicación escolar y evaluar cuidadosamente las adquisiciones académicas, su rendimiento y comportamiento en la escuela o ambientes laborales. Aunque es necesario recordar que los niños con ST, que exhiben dificultades en la escuela a menudo no presentan desórdenes en el aprendizaje, como vimos anteriormente.

Los métodos son los regulares: historia detallada, entrevistas, cuestionarios generales, pruebas y escalas de evaluación, métodos y sistemas de registro.

\subsubsection{Orientación y educación sobre el síndrome}

El foco de la terapia está dirigido a capacitar a la persona con ST, para que cuente con las herramientas necesarias que le permita:

- Enfrentar las dificultades causadas por cada uno de los componentes del trastorno y monitorear el efecto de las terapias.

- Reducir y compensar las desventajas que pueden traer los síntomas del ST, para su adaptación al medio escolar y laboral, según sus reales posibilidades de desempeño,

- Acceder a los diferentes medios de información actualizada sobre el diagnóstico, evolución, tratamiento, pronóstico e investigación del ST; el acceso a bibliografía actualizada sobre distintos temas -"biblioterapia"- ha sido de suma importancia.

- Participar de manera activa para identificar los efectos que la medicación tiene en su caso, según la 
severidad de los síntomas y su discusión con el médico tratante.

- Intervenir en la educación a la comunidad sobre el ST, su prevención y control.

\subsubsection{Terapias dirigidas al abordaje de los diferentes componentes del síndrome y trastornos asociados}

- Terapia conductual de los tics. En muchos casos, son sólo los tics de tipo motor y vocal los síntomas que se presentan como evidencia del síndrome. Tics que pueden ser leves o potencialmente muy aversivos y distrayentes para el niño y su entorno social. No obstante, es importante recordar que la cantidad de ruido que pueden causar no son totalmente voluntarios y que el niño no tiene total control sobre la manifestación de los movimientos y vocalizaciones.

Los tics, como hemos visto, fluctúan en frecuencia, intensidad, duración, localización y modalidad a lo largo del año y tienden a intensificarse conforme la persona se enfrenta a los estresores que se van presentando durante el día. La forma en que el paciente y su familia usan y manejan el lenguaje para referirse a los tics, difiere y depende de las connotaciones familiares y sociales que éstos tengan. En todo caso, siempre es de importancia crucial el saber diferenciar la conducta de la persona. Es la conducta la que describimos y enfrentamos, no la persona.
- Las técnicas cognitivo-conductuales utilizables no han resultado muy exitosas pero pueden ofrecer un soporte realista en los períodos críticos de exacerbación de los tics (King et al., 1999). Éstas son:

- La extinción, basada en el reconocimiento de las señales sensoriales emergentes (la señal es una sensación, un signo extremadamente sutil), las que si son detectadas y rechazadas de manera suficientemente rápida, pueden ser extinguidas sin que se produzca la tensión que amenaza. Lograr el reconocimiento instantáneo de aquel estado no es fácil de alcanzar y requiere de un entrenamiento prolongado e intensivo. La motivación y la persistencia son aliadas indispensables. Cuando se logra la extinción aparente de los síntomas, éstos reaparecerán constantemente, por lo que necesitan ser confrontados y extinguidos hasta el fin. Aunque el resultado es una clase de "media vida" de vigilancia constante y atención dividida.

- La práctica negativa masiva: ejecutar deliberadamente el movimiento por períodos específicos, intercalando por breves momentos períodos de descanso (por ejemplo, cuatro minutos de práctica por uno de descanso). 
Azrin reporta efectividad en la mitad de los estudios en cuanto a la frecuencia, pero no están claros los beneficios a largo plazo. Tuipin revisó 22 estudios y notó que sólo tres mantuvieron el éxito luego de seis meses de seguimiento (King et al., 1999). Muchos otros estudios no reportan cambios en los tics y algunos reportan su incremento. Esta técnica tiene un uso terapéutico limitado.

- Otras técnicas en las que estamos bastante familiarizados los psicólogos son las de manejo de contingencias, en particular las técnicas operantes para el desarrollo, y para la reducción de conductas en ambientes naturales, las técnicas de autocontrol, de entrenamiento en asertividad o habilidades sociales. Si bien es cierto que es difícil generalizar el control a todos los ambientes, es importante señalar los beneficios que trae el uso adecuado de los reforzadores positivos y/o negativos dentro de un plan especialmente elaborado para cada paciente y su familia. No actúan directamente en el aumento o disminución de la frecuencia de los tics pero ayudan a la motivación y, muy especialmente, a la generación de un ambiente coherente, distensionante, sistemático, con metas y límites claros, que facilita la convivencia y evita la creación de interferentes.

- Automonitoreo o autoobservación, con el objetivo real de identificar los estresores, situaciones, etapas, contextos. Es reportado como exitoso (Azrin y Peterson, 1988), principalmente porque el efecto del record inmediato proporciona conciencia de los tics y sus señales.

- Reversión del hábito, que incluye: a) técnicas de registro; b) entrenamiento en el conocimiento de las respuestas (descripción de las respuestas, advertencia, identificación de los signos tempranos y situaciones de generación); c) práctica de respuestas competitivas, como tensar los músculos incompatibles con el movimiento habitual (el que debe sostenerse por varios minutos); d) motivación para el control del hábito, con la revisión de las inconveniencias de ejecutarlo y el reforzamiento positivo por la no ejecución; y e) entrenamiento en la generalización. Los estudios de Azrin y Nunn (1973) señalan la eficacia de las respuestas competitivas, aun cuando éstas no sean similares; por ejemplo, ante el tic de girar el cuello, el contraer la pantorrilla puede resultar exitoso. Miltenberger y Fuqua (1985) señalan 
que la efectividad está en el castigo (la práctica competitiva es experimentada por el sujeto como desagradable). La investigación ha demostrado que los tics son mantenidos por múltiples factores neurobiológicos, como por influencias ambientales y contingencias de reforzamiento (Carr, Taylor, Walander y Reiss, 1996). Esta aproximación sugiere que si ejecutar el tic puede suprimir o reducir el fenómeno sensorial, esta reducción puede servir para reforzar el tic (Evers y Van de Wetering, 1994).

- Tratamiento conductual del Trastorno Obsesivo Compulsivo. Entre los trastornos más vinculados al ST se encuentra el TOC que, se reporta, puede afectar entre el 30\% y $60 \%$ de las personas que presentan el síndrome. Al margen de los fármacos que ayudan notablemente a los que padecen este trastorno, también pueden ser utilizadas las técnicas del comportamiento, las cuales ayudan a manejar la ansiedad y la inquietud, que son resultado de las obsesiones e incluso pueden, en algunos casos, eliminar los comportamientos ritualísticos.

Hay una diferencia entre el desorden obsesivo compulsivo asociado con tics o sin tics. El trastorno sin tics está caracterizado por temores a hacerse daño o hacer daño a algún miembro de la familia; por ejemplo: lavarse las manos se relaciona con prevenir la contaminación; tocar las cosas o personas se vincula con el objetivo de prevenir que ocurra algo terrible, como la muerte de algún familiar. El trastorno con tics es señalado como la urgencia de repetir una actividad hasta que "se sienta suficiente", hasta llegar al "nivel" o a "un arreglo simétrico". Estas urgencias compulsivas no están precedidas típicamente por un temor o dolor, pero pueden estar precedidas por un sentimiento de que algo no está bien o está "incompleto", "como si me estuviera olvidando de algo". Los niños manifiestan experimentar frustración y agitación en su esfuerzo por sentirse bien. Otro problema al que también nos enfrentamos es el de la dificultad para una clara distinción entre compulsión y tics complejos, por lo que hay que definir el síntoma incluyendo los disparadores internos y externos (estímulos discriminativos) para las conductas repetitivas y las urgencias.

En cuanto a las técnicas, la eficacia relativa de la exposición y la prevención de respuesta versus el paradigma de reversión del hábito, necesitan ser estudiadas. Hasta el momento las investigaciones indican que ambas técnicas son deseables.

Los componentes de las técnicas que aparecen como más exitosas 
son la exposición (confrontar el objeto temido) y la prevención de la respuesta (autoinstrucción de no llevar a cabo el ritual o no evitar el estímulo temido). El tratamiento incluye: a) automonitorear e identificar los eventos o situaciones que provocan la urgencia que se va a ritualizar; esto permite al terapeuta y al niño redefinir el vocabulario común sobre los síntomas obsesivocompulsivos; b) evaluar, de acuerdo con la severidad, los estímulos disparadores para ser usados en los ejercicios de exposición y de prevención de la respuesta. Se hacen las gráficas correspondientes. Los instrumentos para realizar esta tarea incluyen la capacitación en el uso de registros narrativos, con los respectivos análisis funcionales de las conductas y el entrenamiento previo en relajación.

Otro conjunto de técnicas lo constituye el manejo de contingencias, que sí se usa como parte de un plan general de tratamiento, definitivamente mejora la confianza y la tolerancia a la ansiedad.

Las técnicas de reestructuración cognitiva y solución de problemas son exitosas cuando los pacientes asumen un rol activo, se encuentran motivados y han logrado habilidad para reportar abiertamente la presencia de rituales y compulsiones (King, 1999). No son recomendables cuando ellos se encuentran de- primidos, tienen pensamiento delusivo acerca de sus obsesiones, y/o la familia es conflictiva. Las técnicas cognitivo-conductuales de los trastornos obsesivo-compulsivos, en combinación con la medicación, en algunos casos, producen una mayor respuesta que cuando se da la medicación sola, hay estudios que reportan una sustancial mejoría e incluso las dos terceras partes de las personas con ST tratadas con técnicas cognitivo-conductuales eran capaces de mantenerse sin medicación dos años después de descontinuarla.

\subsection{Abordaje grupal}

\section{2.1 Con personas que presentan el mismo síndrome}

De esta manera las personas que padecen el síndrome conocen a otras con problemas similares, comparten experiencias, se enteran acerca de los síntomas asociados con los trastornos que padecen, y aprenden otras maneras de enfrentar sus reacciones. El grupo puede proporcionarle una sensación de normalidad y brindarle un ambiente adecuado a la práctica de conductas sociales apropiadas a su condición. El paciente recibe un feedback realístico de sus pares. Los terapeutas pueden monitorear los problemas a fin de intervenir antes de que la situación se salga de control.

Si bien hemos intentado realizar este tipo de terapia, brindándoles a las per- 
sonas con ST un ambiente propicio para el intercambio de opiniones y sensaciones, nos hemos visto frente a dificultades como el denominado "contagio de síntomas", debido principalmente a que muchas personas presentan la tendencia a imitar lo que ven en otros (ecopraxia), lo que oyen de otros (ecolalia) o lo que ellos mismos dicen (palilalia). Tales repeticiones modeladas pueden llevar a la puesta en marcha de nuevos síntomas específicos que van y vienen, y que algunas personas con ST describen como "gatillos" que disparan un tic; por lo que nos estamos limitando a sesiones de dos en dos, en especial en etapas de estabilidad de uno de ellos, de manera que uno sirva de apoyo al otro; ocasionalmente conformamos grupos más amplios. En todo caso la descripción de sus estrategias para el control de sus comportamientos, la expresión de sus logros y dificultades sí han sido efectivas y han influido para la terapia individual. Podemos agregar aquí que las reuniones de carácter social de las familias que conforman los grupos de la Asociación Síndrome de Tourette del Perú, han sido también de mucha ayuda.

\subsubsection{Con otras personas sin el síndrome}

Algunas personas con ST fallan en su intento de obtener éxito para iniciar y mantener relaciones sociales, les es difícil expresar sus ideas y sentimien- tos, comprender y responder adecuadamente a los sentimientos e ideas de otros, reconocer el efecto y consecuencia de su conducta y resolver situaciones de conflicto. Frente a esto, el entrenamiento en habilidades sociales (asertividad), puede ser dado en el hogar, en sesiones terapéuticas y en el propio colegio. La inclusión de este tipo de acción se viene generalizando en las escuelas regulares del medio, lo que favorece la integración de las personas con ST.

\section{TERAPIA Y ORIENTACIÓN EN EL ENTORNO}

\section{Abordaje de la familia}

\subsection{Orientación y educación familiar}

La familia que tiene un miembro que presenta los síntomas del ST, como frente a cualquier otro trastorno crónico, ya viene estresada, frustrada. Los padres pasan por un período "duro" para aceptar los síntomas del síndrome, lo que es propicio para oír su historia y brindarles un espacio crucial para el soporte, el antídoto para la soledad y la desesperación, y también para la culpa que viene asociada por la naturaleza genética del desorden.

Es posible trabajar aquí por reformular la apreciación que se tiene sobre los confusos y emergentes síntomas en el contexto del desorden. Antes del diagnóstico los síntomas son visualizados 
como voluntarios, deliberados, desafiantes, efectos de un escaso control por parte de la persona que los presenta, y la respuesta de la familia es, en general, de recriminación y, en muchos casos, violenta.

Ubicar los síntomas como parte de un trastorno ayuda a discriminarlos, y permite iniciar una discusión más informada sobre el grado de control que puede ser razonablemente esperado respecto de los distintos comportamientos y situaciones. Este diálogo puede romper el círculo vicioso de recriminación-exacerbación de los tics. Ayuda a los padres a focalizarse en la solución del problema antes que en la culpa. Sin embargo, hay que tener cuidado de que esta ubicación de los síntomas no se estigmatice y se conviertan en "parte" de la persona misma.

Son las conductas a las que describimos y enfrentamos, no a la persona. La forma en que la familia y el paciente van a manejar el lenguaje, al referirse a los síntomas, es una parte importante en la exploración, clarificación, comprensión y enfrentamiento del trastorno. No importa cómo se les llame, muchos pueden preferir hablar de hábitos, ruidos, movimientos, manías, rarezas y no usar términos técnicos; eso depende de las connotaciones que cada término tenga para la familia o la comunidad cercana. Inicialmente, en algunas circunstancias no será necesario hablar del término Tourette frente al niño.
En todo caso es de suma importancia cooperar con la familia y la persona con ST, para tal fin es necesario tener en cuenta los siguientes pasos:

- Comprender y familiarizarse con las manifestaciones y episodios del trastorno, brindándoles asistencia para que enfrenten de manera realista sus expectativas.

- Orientar sus reacciones ante las manifestaciones del síndrome, a fin de organizar un ambiente coherente, sistemático, con metas claras, distensionante. En este sentido, será conveniente ayudarlos a:

- Rescatar la visualización de conductas adecuadas antes que las inadecuadas, controlando la dinámica de entrega de los reforzadores condicionados generalizados (atención, aprobación, afecto y reforzadores concretos); y diferenciar las conductas inadecuadas voluntarias de las involuntarias.

- Hacer un uso óptimo de registros $\mathrm{y}$ figuras que visualicen los avances.

- Especificar los efectos o consecuencias de las conductas, haciendo uso de contratos, prioridades y reglas participativas.

- Que apliquen las técnicas operantes, con organización, consistencia y sistematización.

- Enfrentar positivamente su relación con los médicos, otros miembros de la familia, profesores y amigos. 


\subsubsection{Grupos de soporte}

Conformar estos grupos requiere del análisis de variables que son de suma importancia para que se genere la empatía necesaria en ellos y cumplan su verdadera función. Así, se deberá tomar en cuenta la edad de las personas que presentan el síndrome, la severidad del caso y de los trastornos asociados, y como condición secundaria, los niveles culturales y la ubicación geográfica de los miembros que los integran.

Los grupos están dirigidos a manejar el estrés, brindar un espacio para ser escuchados (por personas que comprendan sus frustraciones, temores, molestias y pesares), disminuir sus fantasías o visualizaciones erróneas sobre el síndrome, valorar y visualizar las características de otros miembros del grupo familiar sin ST y analizar la poca atención que generalmente se les da. Es conveniente que estos grupos se reúnan una vez al mes. Existe en internet un grupo de soporte, el que al parecer tiene cierto éxito.

Como se aprecia en la tabla 24 , el $81 \%$ de las familias de los sujetos estudiados iniciaron su participación en los grupos de soporte y se mantienen el $66 \%$ (el $82 \%$ de los que lo iniciaron).
Tabla 24

Participación en grupos de soporte de la ASTP

\begin{tabular}{ccc}
\hline & Iniciaron & Se mantienen \\
\hline & 17 & 14 \\
$\%$ & 80,95 & 66,67 \\
\hline
\end{tabular}

\subsubsection{Terapia familiar}

Ayuda a comprender y evaluar las interacciones entre los miembros de la familia y el impacto de aquellas interacciones, en particular de aquellas que se dan como producto de las manifestaciones de los síntomas. Así, ayudarán a identificar la "condición" y el "rol" de la persona con ST en el seno familiar: si es sobreprotegida, tratada punitivamente, incomprendida, fuente de vergüenza, o si, tal vez, el trastorno domina la interacción familiar. Con fines terapéuticos se pueden detectar los modelos y líderes que operan en el grupo, y evaluar los niveles de aceptación de cada miembro de él.

Todos los miembros aprenden a crear un ambiente familiar más positivo $\mathrm{y}$ efectivo para sostener a la persona con ST y a ellos mismos, y a aceptar al miembro con $\mathrm{ST}$ con sus sintomas, no "a pesar de ellos"; esto proveerá a la persona con ST de un sentido de seguridad para su aproximación saludable al mundo externo. Las esposas de las personas con ST no se percatan del complejo problema del síndrome y sus efectos en las relaciones amorosas hasta después de un tiempo de casados; 
estas reuniones pueden servir para enfrentar estos problemas.

\subsection{Abordaje del entorno social, esco- lar y/o laboral}

\subsubsection{Medio ambiente escolar}

- Educación a maestros y compañeros.Ya que el síndrome no es tan conocido $^{2}$, una de las funciones de los psicólogos tratantes es la de informar a los maestros, colegas y público en general, sobre la naturaleza del síndrome y las maneras como éste afecta la conducta, atención y aprendizaje de las personas con ST, tratando de generar un ambiente propicio de aceptación, sin ridiculización, ya que los síntomas del síndrome pueden ser vistos por los maestros y compañeros como reacciones contra las personas (lo que puede crear consternación, mucha angustia en las personas con ST y sus familias). Los maestros y todo el personal de la escuela pueden reducir el estrés y ayudar a consolidar un ambiente cada vez más positivo. Es de señalar que los maestros van involucrándose con éxito en el manejo de técnicas para la creación y fortalecimiento de habilidades sociales y reso-

2 A pesar de los esfuerzos la Asociación Síndrome de Tourette del Perú hace para difundir sus características. lución de conflictos, que son acciones de suma importancia para el alumno con ST.

- Modificaciones ambientales y académicas. La flexibilidad, comprensión y productividad en el lugar de estudio son de suma importancia. Los estudiantes con ST generalmente tienen los mismos potenciales intelectuales que sus compañeros; sin embargo, como hemos visto, un buen porcentaje de personas con TDAH TDA, TOC y/o ST presentan problemas grafomotores. Los tics pueden interferir la escritura; las personas con TOC pueden presentar compulsiones para escribir, borrar, reescribir palabras, y ordenar las cosas; por lo tanto, puede ser necesario realizar algunas modificaciones en relación con el ambiente académico: instrucción personalizada, tiempo fuera voluntario (para expresar sus tics), exámenes orales, mayor tiempo para los trabajos y exámenes escritos, uso de grabadora, computadora, calculadora; empleo de apoyos visuales (papel cuadriculado o previamente rayado), ubicación especial en el aula, tutoría para el manejo de la agenda o cuaderno de control por el mismo profesor o un compañero, apoyo de compañeros monitores, evaluación de los efectos colaterales de la medicación: somnolencia, tonicidad, entre otros, y según las necesidades. 
- Modificaciones académicas.- Aproximadamente el $25 \%$ de los estudiantes con TDAH, TDA y ST presenta problemas de aprendizaje, un porcentaje mayor que el de 4 a $5 \%$ de la población en general. Otro $25 \%$ presenta problemas significativos relacionados con el trabajo escolar. Los estudiantes con TOC son impactados por las perseveraciones y el estrés. Esta población requiere de educación especializada, entrenamiento y fortalecimiento de las habilidades sociales.

\subsubsection{Medio ambiente laboral}

En ciertos casos es necesario realizar algunas adaptaciones, como acomodamiento al trabajo, acomodamiento de la organización del trabajo, y servicios de apoyo, los que deben ser planteados por la propia persona con ST. La actuación de los terapeutas ocupacionales en este aspecto es de suma importancia.

El psicólogo que asuma la responsabilidad de trabajar con la persona con ST requiere de una preparación especial, profunda, sostenida y comprometida. Su acción requiere de un abordaje integral, no sólo debe dirigirse a la misma persona con ST de manera individual (realizando una evaluación psicológica diferencial y continua, brindando la orientación y educación sobre el síndrome, y aplicando los procedimientos y técnicas de intervención pertinentes según los componentes del síndrome) y en grupos; sino que debe asumir a plenitud su rol de gestor, promotor y de soporte del equipo profesional, del entorno familiar (brindándoles orientación y formación, grupos de soporte y terapia familiar), educativo, social y laboral, responsabilidad que se disfruta cuando se acompaña a la persona con ST a crecer, a que desarrollen sus potencialidades, optimicen su ambiente y actúen de manera competente, confiada en sí misma, en su familia, en la sociedad, buscando alcanzar su plena realización. 


\section{REFERENCIAS}

American Psychiatric Association (1995). Diagnostic and statistical manual of mental disorders. DSM-IV. Washington, 4a. ed.

Azrin, N.H. \& Nunn, R.G. (1973). Habitat reversal: A method of eliminating nervous habits and tics. Behavior Research and Therapy, 11, 619-628.

Azrin, N.H. \& Peterson, A.L. (1988). Habitat reversal for the treatment of the Tourette syndrome. Behavior Research and Therapy, 26, 347-351.

Bados, A. (1999). Los tics y sus trastornos. Naturaleza y tratamiento en la infancia y adolescencia. Madrid: Pirámide.

Bliss, J. (1980). Sensory experiencies of Gilles de la Tourette syndrome. Archives of General Psychiatry, 37, 13451347.

Carr, J.E., Taylor, G.C., Wallander, R.J. \& Reiss, M.L. (1996). A funtional-analytic approach to the diagnosis of a trasient tic disorder. Journal of Behavior Therapy and Experimental Psychiatry, 27, 291-297.

Comings, D.E. \& Comings B.G. (1984). Tourette syndrome and attention deficit disorder. Are they general related. Journal of the American Academy of Child and Adolescent Psychiatric, 23, 148246.

Comings, D.E \& Comings, B.G. (1990). A controlled family history study of Tourette's syndrome. II: Affective and other disorders. J. Clin Psychiatry, 51, 288-291.

Coffey, B., Frazer, J. \& Chen, S. (1992). Comorbidity, Tourette syndrome and anxiety disorders. Advances in Neurology, 58, 95 .

Chee, Ky \& Sachdev, P. (1992). Aust NZ, Psychiatry, 28 (2), 313-318.

Dornbush, M. \& Pruitt, S.H. (1995). Teaching the tiger. A handbook for individuals involved in the education of students with attention deficit disorders, Tourette syndrome or obsessive-compulsive disorder. Hope Press.

Dowling, R, \& Rickler, K. (1986). Tourette syndrome and behavior. Inc. Bayside, Nueva York: TSA INC.

Dowling, R., Cohen, D. \& Leckman, J. (1995). Guide to the diagnosis and treatment of Tourette syndrome. Nueva York: TSA INC.

Evers, R.A.F. \& Van de Wetering, B.J.M. (1994). A treatment model for motor tics based on a specific tension-reduction technique. Journal of Behavior Therapy and Experimental Psichiatry, 25, 255-260.

Filomeno, A. (1994). Algunos problemas de aprendizaje que conciernen al neurólogo. Con mención especial al Síndrome de Tourette. En Montezuma, O. (ed). Revista 25 aniversario del Instituto Santa Magdalena Sofia Barat. Lima. Imprenta SMSB.

Fernández-Álvarez, E. (2002). Trastornos comórbidos relacionados con los tics. Revista de Neurología, 34 (Supl. 1), 122-129.

Haerle, T. (ed.). (1992). Children with Tourette syndrome. A parent's guide. Woodbine house.

Hagin, R. (1993). Tourette syndrome \& the school psychologist. Bayside. Nueva York: TSA INC. 
Head, D., Bolton, D. \& Hymas, N. (1989). Deficit in cognitive shifthing ability with obsessive-compulsive disorder. Biological Psychiatric, 25, 929-937.

King, R., Scahill, L., Findley, D. and Cohen, D. (1999). Psychosocial and behavioral treatments. En: Leckman, J. \& Cohen, D. Tourette's syndrome. Tics, obsessions, compulsions. Chapter 8, 338-358. Nueva York: John Wiley \& Sons, INC.

Kurlan, R., Lichter, D., Hewitt, D. (1989). Sensory tics in Tourette's syndrome. Neurology, 39, 731-734.

Leckman, J., (2002). Introduction to Tourette syndrome \& co-morbid disorders. The self under siege. Conferencia como profesor invitado al curso internacional Síndrome de Tourette y Patología Asociada, organizado por la Asociación Síndrome Tourette del Perú. Lima.

Leckman, J. \& Cohen, D. (1999). Tourette's Syndrome. Tics, obsessions, compulsions. Nueva York: John Wiley \& Sons.

Levi-Pearl, S. (1993). What you should know about tourette syndrome. Nueva York: TSA INC.

Miguel, E. (1995). Phenomenology of intentional repetitive behaviors in obsessive-compulsive disorder and Tourette's disorder. Nueva York: TSA INC.

Miltenberger, R.G. \& Fuqua, R.W. (1985). A comparison of contingent us non contingent competing response practice in the treatment of nervous habit. Journal of Behavior Therapy and Experimental Psychiatry, 16, 195-200.

Montezuma, O. (1998). Guía para maestros de niños con sindrome de tourette.
Lima: Asociación Síndrome de Tourette del Perú.

Robertson, M., Trimble, M. \& Lees, A. (1988). The psychopathology of Gilles de la Tourette Syndrome: A phenomenological Analysis. British Journal of Psychiatry, 152, 383-390.

Rossenberg, L. (1995). Behavior problems and severity of tics. Nueva York: TSA INC.

Scahill, L., Riddle, M.A., Mc SwigginHardin, M., Ort, S.I., King, R.A., Goodman, W.R., Cichetti, D. \& Leckman, J.F. (1997). Children's YaleBrown Tourette Syndrome Association Inc. Questions and Answers about Tourette Syndrome. Bayside, Nueva York: Tourette Syndrome.

Schultz, R.T., Carter, A.S., Gladstone, M., Scahill, L., Leckman, J.F., Peterson, B.S., Zhang, H., Cohen, D.J. \& Pauls, D. (1998). Visual-motor, visuoperceptual and fine motor funtioning in children with Tourette syndrome. Neuropschychology, 12, 134-135.

Shapiro, A. (1989). For the treatment of GTS. Nueva York: TSA INC.

Waltz, M. (2001). Tourette syndrome. Findings answers and getting help. Nueva York: O'Reilly. 